\title{
Protein Sorting in Saccharomyces cerevisiae: Isolation of Mutants Defective in the Delivery and Processing of Multiple Vacuolar Hydrolases
}

\author{
JANE S. ROBINSON, DANIEL J. KLIONSKY, LOIS M. BANTA, AND SCOTT D. EMR* \\ Division of Biology, 147-75, California Institute of Technology, Pasadena, California 91125
}

Received 24 June 1988/Accepted 16 August 1988

\begin{abstract}
Using a selection for spontaneous mutants that mislocalize a vacuolar carboxypeptidase Y (CPY)-invertase fusion protein to the cell surface, we identified vacuolar protein targeting (vpt) mutants in 25 new vpt complementation groups. Additional alleles in each of the eight previously identified vpt complementation groups ( $v p t 1$ through $v p t 8$ ) were also obtained. Representative alleles from each of the 33 vpt complementation groups (vptl through vpt33) were shown to exhibit defects in the sorting and processing of several native vacuolar proteins, including the soluble hydrolases CPY, proteinase A, and proteinase B. Of the 33 complementation groups, 19 were found to contain mutant alleles that led to extreme defects. In these mutants, CPY accumulated in its Golgi complex-modified precursor form which was secreted by the mutant cells. Normal protein secretion appeared to be unafifected in the vpt mutants. The lack of significant leakage of cytosolic markers from the vpt mutant cells indicated that the vacuolar protein-sorting defects associated with these mutants do not result from cell lysis. In addition, the observation that the precursor rather than the mature forms of CPY, proteinase A, proteinase B were secreted from the vpt mutants was consistent with the fact that mislocalization occurred at a stage after Golgi complex-specific modification, but before final vacuolar sorting of these enzymes. Vacuolar membrane protein sorting appeared to be unafiected in the majority of the vpt mutants. However, a subset of the vpt mutants (vpt11, vpt16, vpt18, and vpt33) was found to exhibit defects in the sorting of a vacuolar membrane marker enzyme, $\alpha$-mannosidase. Up to $50 \%$ of the $\alpha$-mannosidase enzyme activity was found to be mislocalized to the cell surface in these vpt mutants. Seven of the vpt complementation groups (vpt3, vpt11, vpt15, vpt16, vpt18, vpt29, and vpt33) contained alleles that led to a conditional lethal phenotype; the mutants were temperature sensitive for vegetative cell growth. This temperature-sensitive phenotype has been shown to be recessive and to cosegregate with the vacuolar protein-sorting defect in each case. Tetrad analysis showed that vpt3 mapped to the right arm of chromosome XV and that vpt15 mapped to the right arm of chromosome II. Intercrosses with other mutants that exhibited defects in vacuolar protein sorting or function ( $v p l, s e c$, pep, and end mutants) revealed several overlaps among these different sets of genes. Together, these data indicate that more than $\mathbf{5 0}$ gene products are involved, directly or indirectly, in the process of vacuolar protein sorting.
\end{abstract}

A basic and fundamental question in biology is that of how a cell is constructed spatially from the information encoded by its genes and from maternally inherited cues and structures. This spatially segregated cellular organization is critical for normal cell functioning. To understand the process, it is important to know not only how the cytoskeletal elements fit together and define the overall shape and structure of the cell but also how proteins, structural and otherwise, find their way to appropriate locations within the cell. An analysis of the assembly and maintenance of each organelle individually could provide a view of the mechanism underlying the cellular construction process.

Protein sorting to mammalian lysosomes represents one of the best-characterized protein delivery pathways $(19,37)$. Much is known about the route that is followed by lysosomal enzymes, and a few components of the sorting machinery have been characterized at the biochemical and molecular levels. However, this is a complex process and we still lack a detailed understanding of the molecular mechanisms underlying this sorting pathway. We chose to work on protein sorting to the yeast equivalent of the mammalian lysosome, the vacuole. The yeast Saccharomyces cerevisiae is the

\footnotetext{
* Corresponding author.
}

organism of choice because we wished to take a genetic approach to solving the vacuolar protein targeting problem. The vacuole is an acidic compartment that is involved in amino acid and inorganic ion storage, as well as various degradative and nutrient recycling functions, especially under starvation conditions $(20,43)$. It contains a variety of hydrolytic enzymes, including the soluble glycoproteins carboxypeptidase $\mathrm{Y}(\mathrm{CPY})$, proteinase $\mathrm{A}(\operatorname{Pr} \mathrm{A})$, and proteinase $B(\operatorname{Pr} B)$, and several membrane-bound enzymes, including an $\alpha$-mannosidase, a proton-translocating ATPase, dipeptidylaminopeptidase $B$, and several permeases $(43,44)$.

Vacuolar proteins transit along with proteins destined for secretion through the early stages of the secretory pathway (38). The transport of the luminal enzymes CPY and PrA has been studied in the greatest detail $(18,18 a, 35 a)$. Each of these enzymes is synthesized initially as an inactive preproenzyme that is translocated into the lumen of the endoplasmic reticulum, where the transient $\mathrm{N}$-terminal signal peptide is removed and the proteins are modified with an $\mathrm{N}$-linked core oligosaccharide (12). These intermediate precursor forms are referred to as p1CPY and p1PrA. The p1 forms are then delivered to the Golgi complex, where further glycosyl modifications convert them to larger $\mathrm{p} 2$ forms. At this stage, it is presumed that proteins on their way to the vacuole are sorted from other secretory proteins destined for 
secretion or assembly into the plasma membrane $(9,11,38)$. Vesicular carriers are presumed to mediate Golgi complexvacuole transfer of these enzymes. At present there is no evidence concerning the existence of an intermediate compartment between the Golgi complex and the vacuole in $S$. cerevisiae analogous to the endosomal compartment detected in mammalian cells. Just prior to, or more likely upon, arrival in the vacuole, the p2 forms of CPY and $\operatorname{PrA}$ are proteolytically cleaved to their mature forms $(22,23)$. This proteolytic maturation is dependent on the presence of active vacuolar $\operatorname{Pr} \mathrm{A}$, and involves the removal of $\mathrm{N}$-terminal prosegments from each protein (13). Similarly, other vacuolar enzymes such as PrB also appear to be made and transported in their precursor forms and to undergo $\operatorname{PrA}$ dependent proteolytic maturation in the vacuole $(21,22 ; \mathrm{C}$. Moehle, C. K. Dickxon, and E. Jones, J. Cell Biol., in press).

The observations that mutations in the CPY vacuole sorting signal or high gene dosage-induced overproduction of CPY and PrA lead to missorting and secretion of these proteins indicate that a failure to sort vacuolar proteins results in their delivery to the cell surface $(15,39,41)$. This rationale was used by Rothman and Stevens (35) to identify mutants that were defective in vacuolar protein sorting by isolating yeast strains that secreted CPY. Through this approach, they identified mutants that were assigned to eight complementation groups (referred to as $v p l$, for vacuolar protein localization-defective mutants). Independently, we used a gene fusion-based selection scheme to isolate mutants defective in vacuolar protein targeting (vpt) (2). By this approach, mutants defective in the vacuolar sorting of a CPY-invertase (Inv) hybrid protein (CPY-Inv433) (15) were selected. Eight $v p t$ complementation groups were identified and shown to missort and secrete the CPY-Inv hybrid protein as well as the wild-type CPY protein (2). The advantages of the Inv fusion approach include the following. (i) The identification of mutants is not dependent on their secretion of active CPY; (ii) the gene fusion approach, in theory, could be applied to any vacuolar protein whether its biochemical activity was known or not; and (iii) the absence or presence of external Inv activity can be easily assayed, and powerful genetic selection procedures have been developed that demand the presence of external Inv activity.

In this study we greatly extended the gene fusion approach to isolate and biochemically characterize some 500 new vpt mutants. Here we describe the genetic and biochemical analysis of these vacuolar protein-targeting mutants. Our results indicate that many different gene functions participate in the events associated with normal vacuolar protein sorting. This process is likely to involve several distinct steps, including the selective recognition of vacuolar proteins, their packaging into transport vesicles, delivery of these vesicles to the correct target organelle, recognition and fusion with the target, release of the vacuolar proteins, and recycling of transport components for additional rounds of protein sorting.

\section{MATERIALS AND METHODS}

Yeast strains. $S$. cerevisiae SEY6210 (MAT $\alpha$ leu2-3,112 ura3-52 his3- 4200 trpl- $\Delta 901$ lys2-801 suc2- $\Delta 9$ GAL) and SEY6211 (MATa leu2-3,112 ura3-52 his3- $\Delta 200$ trpl- $\Delta 901$ ade2-101 suc2- $\triangle 9 G A L$ ) were constructed by standard genetic methods (36). Both strains contained a complete deletion $(s u c 2-\Delta 9)$ of the chromosomal copy of $S U C 2$ and contained no other unlinked Inv structural genes (7).
SEY6210 and SEY6211 were transformed to uracil independence with either of two CPY-Inv fusion-encoding plasmids, pCYI-50 and pCYI-433 (15). vpt mutant alleles vptl-1 through vpt8-I (2) in the SEY2101 (MATa ura3-52 leu2-3,112 suc2- $\Delta 9$ ade2-101) (7) strain background were used for complementation tests with new vpt mutants isolated in strain SEY6210 (this study). They were also crossed with strain SEY6210 to give MAT $\alpha$ lys2-801 vpt $1-1$ through vpt8-1 strains for crossing with new vpt mutants isolated from SEY6211.

Media and genetic methods. YPD medium, minimal yeast medium, sporulation medium, and bromocresol purple indicator plates were prepared as described previously (36). The bromocresol purple plates contained $2 \%$ sucrose as the sole carbon source and were spread with $0.2 \mathrm{mg}$ of antimycin A in $95 \%$ ethanol just before use (2). The minimal medium of Wickerham (42), which was modified as described previously (15), was used for cell growth before and during labeling with $\mathrm{Na}^{35} \mathrm{SO}_{4}$ (ICN Radiochemicals). Standard genetic methods (36) were used throughout this study. Replica plating to test phenotypes of segregants was done with a 48-pronged replicator by using dilution in sterile 96-well microtiter dishes (24). Rapid complementation analysis was carried out by using the pronged replicator to transfer arrays of 48 new vpt mutants to microtiter dishes containing $v p t$ tester strains of the opposite mating type suspended in liquid YPD medium. Mating and diploid growth were allowed to occur in the wells for 2 days at $26^{\circ} \mathrm{C}$. Replicas were then made onto minimal medium, which was supplemented to select for diploid strains. The resulting patches of diploid strains were transferred without dilution to YP fructose and assayed for external Inv activity by using a rapid filter replica assay method $(15,18)$ to score complementation.

Enzyme assays. The procedures and unit definitions used for assays of invertase and $\alpha$-glucosidase (15), CPY (39), PrA (1), $\alpha$-mannosidase (28), and glyceraldehyde-3-phosphate dehydrogenase (Worthington Enzyme Manual; Worthington Diagnostic, Freehold, N.J.) (3) have been described previously.

Labeling, fractionation, and immunoprecipitation. Spheroplasts were prepared and labeled by a modification of a previously described method (32). Cells were grown to the mid-log phase in the minimal medium of Wickerham (42) that was supplemented with $0.2 \%$ yeast extract. Four units of cells at an optical density at $600 \mathrm{~nm}$ were then centrifuged and suspended in $0.1 \mathrm{M}$ Tris sulfate $(\mathrm{pH} 9.4)-10 \mathrm{mM}$ dithiothreitol and incubated at $30^{\circ} \mathrm{C}$ for $5 \mathrm{~min}$, centrifuged again, and suspended in the minimal medium of Wickerham (42) that was adjusted to $\mathrm{pH} 7.4$ and that contained $1.3 \mathrm{M}$ sorbitol. Lyticase was added to a final activity of $30 \mathrm{U}$ per optical density unit at $600 \mathrm{~nm}$, and the cultures were incubated at $30^{\circ} \mathrm{C}$ for $20 \mathrm{~min}$ to remove the cell wall. Spheroplasts were pelleted, suspended in the minimal medium of Wickerham (42) containing $1.3 \mathrm{M}$ sorbitol, and labeled with $\operatorname{Tran}^{35} \mathrm{~S}$ label $(0.2 \mathrm{mCi} / \mathrm{ml})$ for $20 \mathrm{~min}$ at $30^{\circ} \mathrm{C}$. Chase was initiated by the addition of methionine (final concentration, $50 \mu \mathrm{g} / \mathrm{ml}$ ). The labeled culture was separated into spheroplast (intracellular) and medium (extracellular) fractions. To examine proteins in the medium fraction, cells were grown and labeled as described previously (15). The whole cells were then separated from the medium fraction by centrifugation, precipitated with $5 \%$ trichloroacetic acid, suspended in loading buffer, and run on sodium dodecylsulfate-10\% polyacrylamide gels. Antisera to $\operatorname{PrA}, \mathrm{CPY}$, and Inv were prepared as described previously (18a).

Materials. Lyticase was obtained from Enzogenetics, 
$\operatorname{Tran}^{35} \mathrm{~S}$ label was from ICN Radiochemicals and all other chemicals were from Sigma Chemical Co. (St. Louis, Mo.). Antiserum to PrB was a gift from C. Moehle and E. Jones.

\section{RESULTS}

Isolation of 25 new vpt complementation groups. Previously, we observed that short amino-terminal domains of the CPY sequence, when fused to mature enzymatically active Inv, can quantitatively divert delivery of this normally secreted enzyme to the yeast vacuole (15). Yeast cells require external Inv activity to grow on sucrose as a sole fermentable carbon source, so we could exploit the sorting behavior of the CPY-Inv hybrid protein to select mutants that missort and secrete the fusion protein. Yeast strains deleted for all endogenous genes encoding invertase $(\Delta s u c)$ but containing a low-copy-number (CENIV, ARSI) plasmid encoding an appropriate CPY-Inv hybrid protein as their only source of Inv activity could not grow on sucrose, because all the Inv activity was sequestered in the vacuole (Fig. 1). Suc ${ }^{+}$derivatives of such cells can be isolated simply by selecting for growth on sucrose-containing medium. Based on earlier results, many of these mutants were expected to mislocalize the native vacuolar enzyme CPY as well, because targeting of the fusion proteins to the vacuole is dependent on the presence of the CPY vacuolar sorting signal at the amino-terminal end of the CPY-Inv hybrid proteins (15). In the present study, we decided to select such mutants using two different CPY-Inv hybrid proteins: CPYInv50 (encoded by plasmid pCYI-50) and CPY-Inv433 (encoded by plasmid pCYI-433) (Fig. 1). These gene fusions were composed of the coding sequence for the N-terminal 50 and 433 amino acids of proCPY, respectively, which were fused in-frame to a truncated form of the $S U C 2$ gene carried on the Escherichia coli-S. cerevisiae shuttle vector pSEYC306 (15). This modified SUC2 gene lacked its 5'regulatory sequences and the coding sequence for its aminoterminal signal peptide (15) (Fig. 1).

$\mathrm{Suc}^{+}$mutant strains were isolated from the $\Delta s u c 2$ strains SEY6210 and SEY6211 harboring either pCYI-50 or pCYI433. To select $\mathrm{Suc}^{+}$mutants, single colonies of the four parental strains were plated onto YP medium with sucrose as the sole carbon source, antimycin $A$ as an inhibitor of respiration, and bromocresol purple as a $\mathrm{pH}$ indicator; bromocresol purple turns yellow when sucrose is being fermented. Only one spontaneous mutant derived from each parental colony was picked, to ensure that each mutation event was independent. A total of $505 \mathrm{Suc}^{+}$mutants were obtained and characterized: $241 \mathrm{MATa} \mathrm{Suc}^{+} v p t$ mutants (isolated from SEY6211) and 264 MAT $\alpha \mathrm{Suc}^{+} v p t$ mutants (isolated from SEY6210). We confirmed that these vpt mutants secreted the CPY-Inv hybrid protein by carrying out a rapid filter assay for external Inv activity. This assay involved patching the mutants onto YP plates containing fructose, incubating the plates at $26^{\circ} \mathrm{C}$ for 24 to $36 \mathrm{~h}$, and then making replicas onto Whatman no. 1 filter papers (Whatman, Inc., Clifton; N.J.) that were presoaked in an Inv assay solution $(15,18)$. External Inv reacted with the assay mixture to produce a red spot at the position of the appropriate cell patch. The presence of internal Inv activity was confirmed by exposing the cell patches to chloroform vapor, which lyses the yeast cells, prior to transfer onto the assay filter. This simple method permitted us to get an initial indication of the extent of the mislocalization defect for each new $v p t$ mutant. All of the $v p t$ mutants were found to express external Inv activity by this assay. In the parental control
A

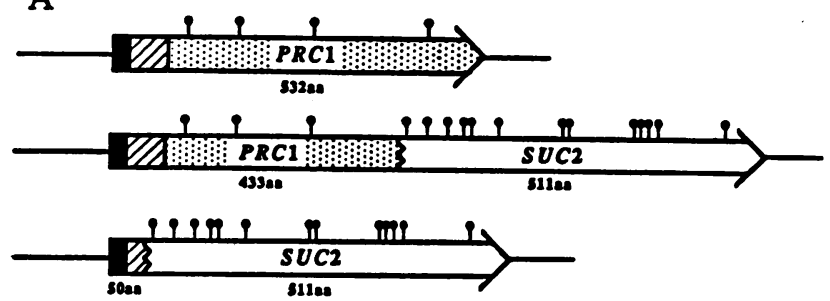

B

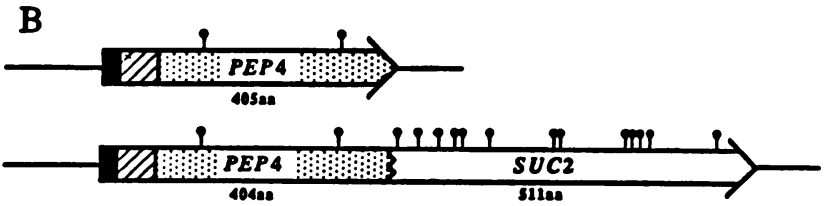

C

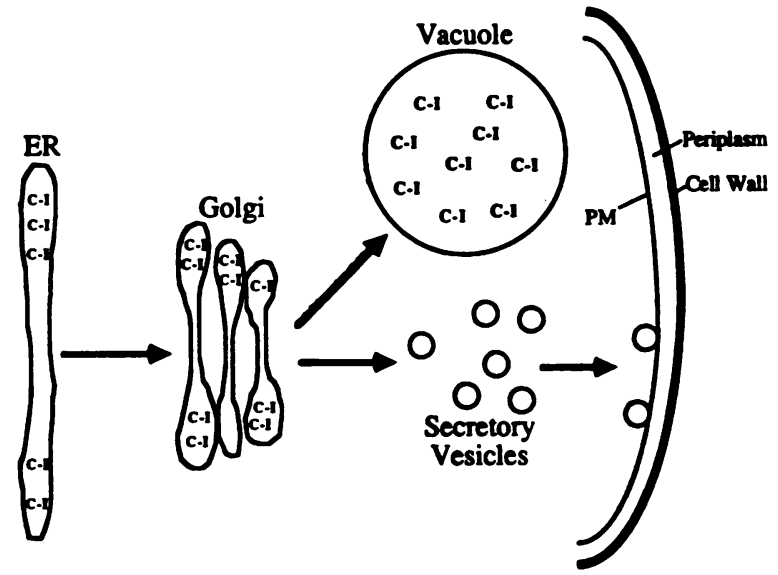

FIG. 1. Selection scheme for $v p t$ mutants. (A) PRCI-SUC2 gene fusions encoding CPY-Inv hybrid proteins. At the top is a schematic representation of the gene encoding CPY $(P R C 1)$. The signal sequence is indicated by a solid black box, the prosegment is indicated by hatched lines, and the mature sequences are indicated by dots (15). The positions of the oligosaccharide addition sites are indicated by tadpole-shaped forms. The two fusions of $P R C I$ sequences to $S U C 2$ used to select $v p t$ mutants are shown below. The 50-aminoacid fusion received extensive glycosyl modification, whereas the 433-amino-acid fusion did not become hyperglycosylated. (B) The gene encoding PrA (PEP4) and a representation of the PEP4-SUC2 gene fusion tested for its localization in the vpt mutants (Table 1). (C) A simple diagram showing the vacuolar protein delivery route followed by the CPY-Inv hybrid protein (C-I). The vpt mutants were selected for their mislocalization of some or all of this CPY-Inv hybrid protein to the cell surface. For some of the vpt mutants, secretion of the mislocalized CPY-Inv was shown to be dependent on $S E C I$ gene function, indicating that delivery to the surface is mediated by secretory vesicles (2). ER, Endoplasmic reticulum; aa, amino acid; PM, plasma membrane.

strains, all of the Inv activity was internal, as determined by the assay.

The Inv filter assay was also used to determine whether the vpt mutations were dominant or recessive in diploid strains derived by backcrossing each of the $v p t$ mutants to the appropriate parental strain (SEY6210 or SEY6211). Recessive vpt mutants were then intercrossed, diploid strains were isolated, and the filter assay was used to assign the MATa $v p t \times M A T \alpha v p t$ strains to complementation groups (Fig. 2). Complementing pairs of recessive $v p t$ mutants exhibited no external Inv activity (white patch on Inv filter assays), whereas noncomplementing pairs of $v p t$ mutants gave rise to red patches on the filters corresponding to 


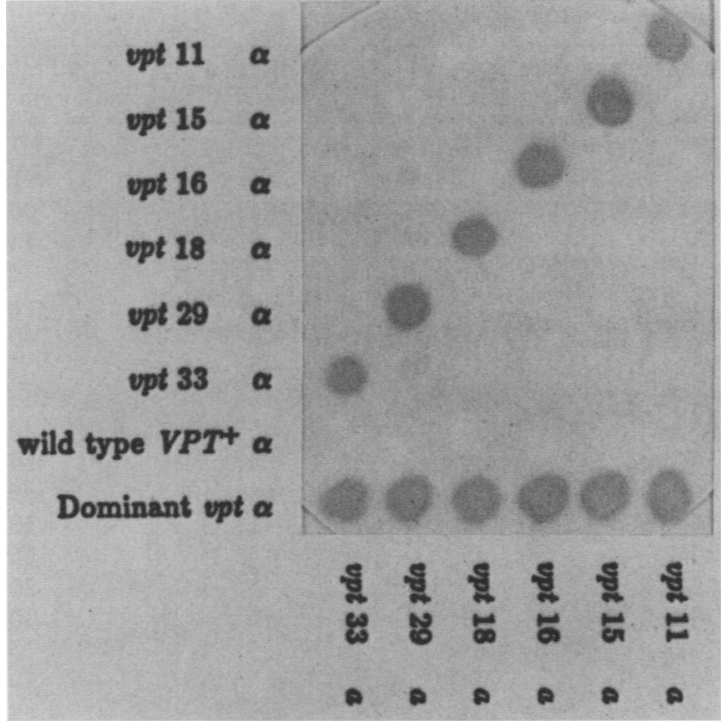

FIG. 2. Complementation analysis of $v p t$ mutants. Secreted Inv activity of intact cells. Diploids were constructed by crossing $v p t$ MATa lys2 with vpt MATa ade2 mutants and selecting Ade ${ }^{+}$Lys $^{+}$ diploid strains on minimal medium. These diploid strains were patched in an array onto YP medium containing fructose and screened by the Inv filter assay. Noncomplementation was seen as a red spot, indicating secretion of the CPY-Inv hybrid protein, while complementation was observed as no reaction (white).

the secretion of Inv activity. In general, the assay gave unambiguous results, as shown in Fig. 2. Of the 505 new vpt mutants, 17 were found to contain mutations that led to a dominant $\mathrm{Suc}^{+}$phenotype. Of six of these dominant mutants tested, only one was found to harbor a mutation linked to the fusion plasmid. The other mutants apparently contained dominant chromosomal mutations. These have not yet been characterized further. The remaining 488 mutants contained recessive mutations. Of these, 184 (84 MATa and 100 MAT $\alpha$ ) could be assigned to the previously identified $v p t$ complementation groups (vptl through vpt8) (2). The remaining MATa vpt mutants were systematically crossed with new MATa vpt mutants until they could be assigned to new complementation groups (arbitrarily defined as containing one MATa and one MAT $\alpha$ allele). Twenty-five new $v p t$ complementation groups were identified ( $v p t 9$ through $v p t 33$ ) (Table 1). Mutant alleles in each of the 33 complementation groups identified were obtained with both the pCYI-50 and the pCYI-433 fusion plasmids. A total of 21 recessive MATa $v p t$ mutants and 36 recessive $M A T \alpha$ vpt mutants were crossed with at least one allele from each of the $33 v p t$ complementation groups and, in addition, were crossed with all the remaining unassigned $v p t$ mutants of the opposite mating type. All 21 unassigned MATa mutants complemented each of the remaining 36 unassigned MATa $v p t$ mutants. We did not construct MATa and MAT $\alpha$ alleles of each of these 57 unassigned vpt mutants and continue the complementation analysis further.

Because the vpt mutants were selected as spontaneous events, we expected that they would correspond to singlesite mutations. To obtain further support of this, many of the vpt mutants (vpt3, vpt4, vpt7, vpt9, vpt11, vpt14, vpt15, $v p t 16, v p t 18, v p t 29$, and vpt33) were crossed with parental $V P T$ strains and subjected to tetrad analysis. For each mutant tested, the vpt defect and all the other markers scored in the cross segregated with the expected 2:2 Men- delian inheritance pattern of simple single-locus mutations. This confirmed that these vpt mutants represent single-site changes and further indicated that the genetic defects associated with these mutants do not result in any gross mating or chromosomal abnormalities. In the course of these analyses, we noticed that two of the vpt mutants appeared to be linked to auxotrophic markers that were heterozygous in the crosses. The segregation data for $v p t 3$ and ade2 (17 parental ditype: 1 tetratype:0 nonparental ditype) indicated that $v p t 3$ was linked to ade2. The approximate map distance (calculated as described previously [36]) between ade2 and vpt3 was $\sim 2.8$ centimorgans. This locates the VPT3 locus on the right arm of chromosome $\mathrm{XV}$, where $A D E 2$ has been mapped (25). Another of the vpt mutants, vpt15, appeared to be linked to lys2 (17 parental ditype:5 tetratype:0 nonparental ditype), in this case less tightly ( $\sim 11.4$ centimorgans). The LYS2 gene has been mapped to the right arm of chromosome II (25).

Mislocalization of vacuolar enzymes in the vpt mutants. At least one mutant from each of the $33 \mathrm{vpt}$ complementation groups was subjected to a series of enzyme assays to quantitate the extent of the mislocalization defect and to test whether mislocalization of the CPY-Inv fusion protein was due to cell lysis.

The extent of mislocalization of the CPY-Inv hybrid protein was determined for several alleles of each complementation group by assaying external Inv (in whole cells) as well as total Inv (in lysed cells) and then calculating the percentage of Inv activity that was secreted (15). The results obtained for a representative vpt mutant allele from each complementation group are presented in Table 1 . In most cases other alleles from the same complementation group secreted the CPY-Inv hybrid protein to a similar extent. Two to five alleles from each vpt complementation group were assayed. The alleles for which assay data are shown in Table 1 were used in all subsequent analyses.

The most obvious unrelated defect that might lead to the apparent secretion of vacuolar proteins is cell lysis. For this reason, assays of a cytoplasmic marker enzyme, glyceraldehyde-3-phosphate dehydrogenase, were carried out to determine whether any of the $v p t$ mutants exhibited significant levels of external glyceraldehyde-3-phosphate dehydrogenase activity. In no case was there evidence of significant leakage of this cytoplasmic marker enzyme into an extracellular fraction. Furthermore, the level of cell-associated glyceraldehyde-3-phosphate dehydrogenase activity detected after detergent lysis of vpt mutant cells was similar to that detected in the parental strains (SEY6210 and SEY6211) (Table 1). In addition, the mutants were tested for the presence of external $\alpha$-glucosidase, another cytoplasmic enzyme. Again, no evidence for lysis was found (data not shown). As discussed below, the vpt mutants secreted precursor forms of the vacuolar proteins rather than the mature forms, as would have been expected if cell lysis were responsible for the observed secretion.

As an initial crude test to determine whether the new $v p t$ mutants also secrete CPY, we assayed CPY enzyme activity in each mutant. Since CPY is normally processed from the inactive $\mathrm{p} 2$ zymogen to the mature, active form inside the vacuole, it is expected that if CPY is missorted into the media, it will not become activated or will be activated poorly $(38,39)$. Thus, the more extreme the $v p t$ mislocalization defect, the lower the cell-associated CPY activity is expected to be relative to the activities of the parental strains SEY6210 and SEY6211. CPY activity levels ranged from $2 \%$ of parental activity in $v p t 5$ up to $82 \%$ in $v p t 23$ (Table 1). CPY 
TABLE 1. vpt complementation groups and enzyme assay data ${ }^{a}$

\begin{tabular}{|c|c|c|c|c|c|c|c|}
\hline $\begin{array}{l}\text { Complementation } \\
\text { groups }\end{array}$ & $\begin{array}{l}\text { Total no. } \\
\text { of alleles }\end{array}$ & $\begin{array}{l}\text { No. of } \\
t s \text { alleles }\end{array}$ & $\begin{array}{l}\% \text { CPY-Inv } \\
\text { secreted }\end{array}$ & $\begin{array}{l}\text { \% of parent } \\
\text { CPY activity }\end{array}$ & $\begin{array}{l}\% \text { PrA-Inv } \\
\text { secreted }\end{array}$ & $\begin{array}{l}\text { \% External } \\
\text { G3PDH }\end{array}$ & $\begin{array}{c}\% \text { External } \\
\alpha \text {-mannosidase }\end{array}$ \\
\hline$v p t 1$ & 46 & & 85 & 10 & 45 & 5 & $<5$ \\
\hline$v p t 2$ & 29 & & 25 & 65 & ND & ND & ND \\
\hline$v p t 3$ & 11 & 1 & 80 & 10 & 75 & $<5$ & $<5$ \\
\hline vpt4 & 15 & & 75 & 5 & 65 & $<5$ & $<5$ \\
\hline$v p t 5$ & 9 & & 100 & $<5$ & 65 & $<5$ & 5 \\
\hline vpt6 & 6 & & 70 & 5 & 45 & $<5$ & $<5$ \\
\hline$v p t 7$ & 32 & & 90 & 30 & 55 & $<5$ & 10 \\
\hline vpt8 & 33 & & 65 & 25 & 65 & $<5$ & $<5$ \\
\hline$v p t 9$ & 20 & & 65 & 35 & 55 & $<5$ & $<5$ \\
\hline vpt 10 & 29 & & 50 & 30 & 35 & $<5$ & 20 \\
\hline vptll & 8 & 2 & 90 & 10 & 60 & $<5$ & 25 \\
\hline vpt12 & 3 & & 65 & 70 & 55 & $<5$ & $<5$ \\
\hline vptl3 & 26 & & 100 & 25 & 110 & $<5$ & 10 \\
\hline vpt 14 & 8 & & 50 & 45 & 35 & $<5$ & 15 \\
\hline vpt15 & 14 & 8 & 80 & 25 & 65 & 5 & 20 \\
\hline vptl6 & 5 & 2 & 75 & 10 & 60 & $<5$ & 30 \\
\hline vpt17 & 40 & & 80 & 20 & 65 & $<5$ & $<5$ \\
\hline vpt 18 & 8 & 3 & 100 & 30 & 90 & $<5$ & 30 \\
\hline vpt19 & 16 & & 80 & 20 & 100 & $<5$ & $<5$ \\
\hline vpt 20 & 5 & & 35 & 75 & 30 & 5 & 5 \\
\hline vpt 21 & 2 & & 85 & 45 & 105 & $<5$ & 5 \\
\hline vpt 22 & 4 & & 35 & 50 & 25 & $<5$ & $<5$ \\
\hline vpt 23 & $\therefore$ & & 25 & 80 & 30 & $<5$ & $<5$ \\
\hline vpt 24 & 8 & & 40 & 50 & 50 & $<5$ & 10 \\
\hline vpt 25 & 4 & & 35 & 50 & 30 & 5 & $<5$ \\
\hline vpt 26 & 4 & & 95 & 15 & 70 & $<5$ & 15 \\
\hline vpt 27 & 9 & & 40 & 40 & 40 & $<5$ & 20 \\
\hline vpt28 & 7 & & 35 & 65 & 40 & $<5$ & 5 \\
\hline vpt 29 & 8 & 2 & 70 & 15 & 90 & $<5$ & 10 \\
\hline$v p t 30$ & 4 & & 60 & 40 & 70 & $<5$ & 5 \\
\hline vpt31 & 2 & & 15 & 65 & 30 & $<5$ & 5 \\
\hline vpt32 & 5 & & 35 & 55 & 35 & $<5$ & 5 \\
\hline$v p t 33$ & 7 & 5 & 90 & 10 & 85 & $<5$ & 20 \\
\hline VPT $^{+}$ & & & 5 & 100 & 5 & $<5$ & $<5$ \\
\hline
\end{tabular}

${ }^{a}$ The vpt mutants were assigned to 33 complementation groups on the basis of standard complementation analysis. Also shown is the number of temperature-sensitive vpt mutants in each of the seven complementation groups that had Ts alleles. Inv assays were performed on several alleles of each complementation group. Whole cells were assayed for external Inv activity, and lysed cells were assayed for total Inv activity. The percentage of external Inv activity was calculated for each mutant allele. A strongly defective allele (high extracellular percentage of Inv) was chosen for further analysis. Levels of other enzyme activities secreted by, or associated with, these representative alleles are presented. Abbreviations: G3PDH, glyceraldehyde-3-phosphate dehydrogenase; ND, not determined.

activities were inversely proportional to the level of the CPY-Inv hybrid protein secreted from most of the vpt mutants (Table 2). For example, some mutants such as vpt5 and $v p t 33$ were very defective by both criteria, whereas others, including $v p t 2$ and $v p t 20$, did not show major defects by either assay. This result indicates that the behavior of the CPY-Inv hybrid protein in the vpt mutants accurately reflects the effect that these mutations have on CPY activity and, presumably, on its localization.

The availability of PrA-Inv hybrid proteins that were efficiently targeted to the vacuole (Fig. 1) $(18,18 \mathrm{a})$ offered a simple way for us to test the effect of $v p t$ mutations on the localization of another protein that is found in the vacuole in wild-type yeast. To this end, the representative $v p t$ alleles were cured of the plasmids encoding CPY-Inv fusions and retransformed with a vector ( $\mathrm{pP} 4 \mathrm{I}-404)$ encoding a fusion protein, PrA-Inv404, consisting of the N-terminal 404 amino acids of PrA fused to mature active Inv. The hybrid protein expressed by pP4I-404 is delivered efficiently to the vacuole in $S$. cerevisiae VPT strains (18a). Inv assays showed that in most of the vpt mutants significant amounts of PrA-Inv hybrid protein were secreted instead of being targeted to the

TABLE 2. Grouping of $v p t$ mutants based on CPY and CPY-Inv localization ${ }^{a}$

\begin{tabular}{|c|c|c|c|c|}
\hline \multirow{2}{*}{$\begin{array}{c}\% \text { of CPY-Inv } \\
\text { activity secreted }\end{array}$} & \multicolumn{4}{|c|}{ vpt complementation group with the following CPY activities (\% of parent): } \\
\hline & $<15 \%$ & $15-30 \%$ & $30-50 \%$ & $>50 \%$ \\
\hline$>90 \%$ & $5,26,33$ & $7,13,18$ & & \\
\hline $65-90 \%$ & $1,3,4,6,11,16$ & $15,17,19,29$ & 21 & \\
\hline $40-65 \%$ & & 8,10 & 9, 30, 14, 24 & 12 \\
\hline$<40 \%$ & & & 27 & $2,20,22,23,25,28,31,32$ \\
\hline
\end{tabular}

${ }^{a}$ Grouping of $v p t$ mutants based on severity of CPY and CPY-Inv fusion protein localization defects. The percentage of CPY enzyme activity was broken into four levels, as was the percentage of CPY-Inv hybrid protein secreted. The $33 v p t$ complementation group numbers are shown in the appropriate spaces. vpt mutants that showed severe mislocalization defects, processing defects, or both types of defect for both CPY and PrA, as shown by cell fractionation and immunoprecipitation of these proteins (Fig. 3), are given in boldface. 


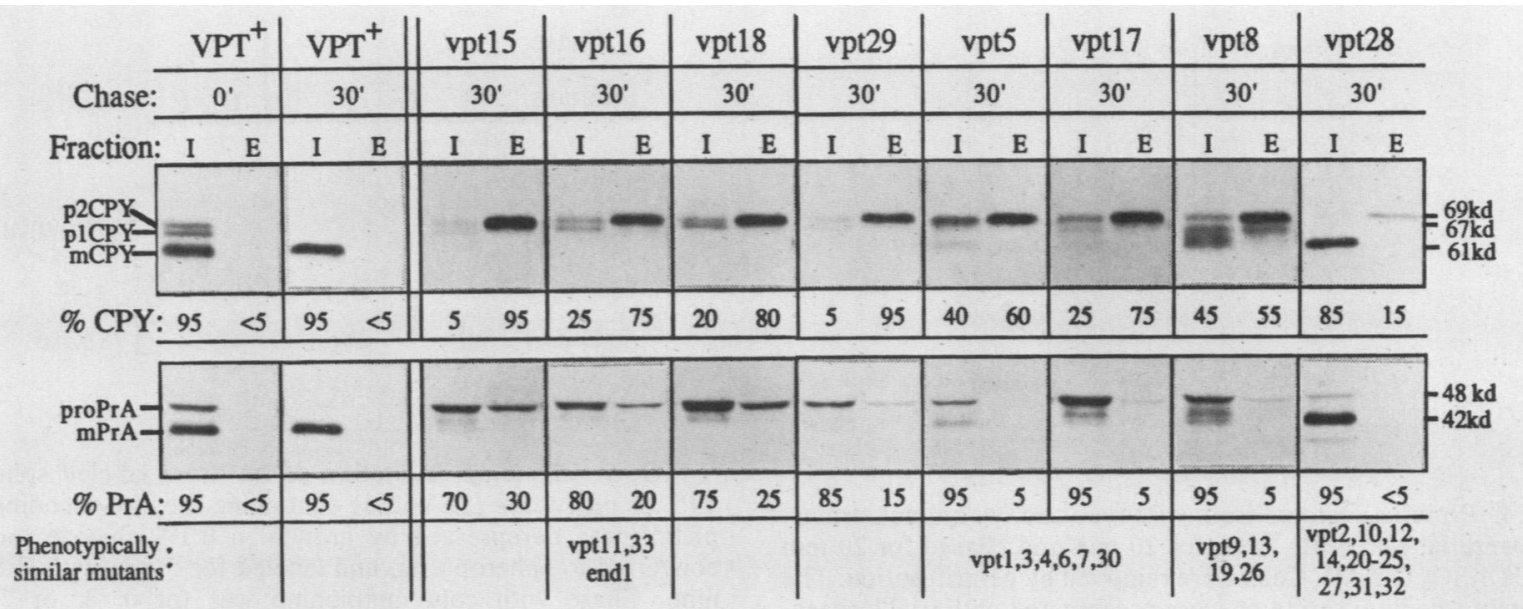

FIG. 3. Immunoprecipitation of CPY and PrA in wild-type (SEY6210 and SEY6211) and vpt mutant yeast strains. Spheroplasts were labeled (as described in the text) and chased for the indicated times (in minutes). The spheroplast pellet made up the internal (I) fraction and the supernatant constituted the external (E) fraction in each case. Immunoprecipitations were carried out with CPY or PrA antisera. Densitometer tracings were used to calculate the approximate percentages of CPY and PrA in the internal and external fractions for each mutant shown. The vpt mutants with similar phenotypes to those shown are listed. Distribution patterns of CPY and PrA for vptl, vpt 3 , vpt4, $v p t 6, v p t 7$, and $v p t 30$ ranged between those shown for $v p t 5$ and $v p t 17$. The migration positions of precursor and mature forms of CPY (mCPY) and $\operatorname{PrA}(\mathrm{mPrA})$ and their approximate molecular sizes (in kilodaltons [kd]) are indicated. For many of the mutants a protein form intermediate in size between p2CPY and mature CPY was immunoprecipitated by anti-CPY antisera. This band migrated at approximately the same position as p1CPY on our gels. The p1 and p2 forms of PrA could not be resolved under the gel conditions used.

vacuole (Table 1). The most defective vpt mutants, as estimated by the reduction in CPY activity levels and secretion of the CPY-Inv hybrid proteins, were also the mutants that exhibited the largest defects in PrA-Inv sorting (Table 1). It is possible that both PrA and CPY use a common delivery pathway.

We were also interested in determining whether, in addition to the sorting of soluble vacuolar hydrolases, sorting of vacuolar membrane proteins was affected in any of the $v p t$ mutants. One vacuolar membrane enzyme that has been well characterized is $\alpha$-mannosidase. This enzyme is not dependent on PrA processing for its activation (17), and thus would be expected to remain active even when it was mislocalized (to the cell surface, for example). The vpt mutants were tested for the presence of cell surface $\alpha$ mannosidase activity by assaying whole, as well as detergent-lysed, cells. In all of the $v p t$ mutants, total $\alpha$-mannosidase activity levels were comparable to that of the wild type $(V P T)$. The only vpt mutants that showed significant levels of external cell-associated $\alpha$-mannosidase activity were vpt10, vpt11, vpt15, vptl6, vpt18, vpt27, and vpt33 (Table 1). It is interesting that these mutants, with the exception of vpt10 and vpt27, were severely defective by several other criteria, including CPY and PrA mislocalization (Fig. 3), and the possession of additional phenotypes such as temperature sensitivity (Table 1) and abnormal vacuolar morphology (L. M. Banta, J. S. Robinson, D. J. Klionsky, and S. D. Emr, J. Cell. Biol., in press). All of the representative $v p t$ alleles shown in Table 1 were non-temperature-sensitive alleles. To further address the $\alpha$-mannosidase localization defect, we also assayed the potentially more severe temperature-sensitive (Ts) alleles of each complementation group for which they were available (vptll, vpt15, vpt 16, vpt 18, and vpt33). Interestingly, we observed as much as 40 to $50 \%$ of the $\alpha$-mannosidase activity at the cell surface in some Ts alleles of vpt11, vpt16, vpt18, and $v p t 33$, even when the cells were grown at the permissive growth temperature $\left(25^{\circ} \mathrm{C}\right)$. No evidence for cell lysis of the Ts alleles was obtained.
We suspected that some of the vpt mutants might secrete an altered spectrum of proteins compared with that of wild-type $S$. cerevisiae for two reasons. First, since secretory and vacuolar proteins transit together through portions of the secretory pathway (38), some gene products affecting the delivery of molecules to the vacuole might also affect the transport of other molecules destined for secretion. Thus, if the vpt mutations affected the secretion pathway, some extracellular proteins might be missing or reduced in the medium isolated from certain of the vpt mutant strains. Second, and conversely, additional protein species may accumulate in the growth medium, since the vpt mutants were selected for their ability to secrete vacuolar contents. To address these questions, we examined the secretion of radioactively labeled proteins by some $v p t$ mutants into the growth medium. Cells were labeled with ${ }^{35} \mathrm{SO}_{4}$ for 20 min and then chased for $20 \mathrm{~min}$ in the presence of excess unlabeled $\mathrm{SO}_{4}$. Media proteins were precipitated in trichloroacetic acid, resolved by sodium dodecyl sulfate-polyacrylamide gel electrophoresis, and examined by autoradiography (Fig. 4). No striking differences between the media fractions isolated from wild-type or $v p t$ mutant cells were observed. Comparable amounts of a similar set of proteins were observed in extracts from both wild-type and $v p t$ mutant cells. Two of the mutants (vpt7 and vptll) exhibited an extra protein band, in addition to the set observed in VPT $S$. cerevisiae (Fig. 4). We did not determine whether either of these new bands corresponded to a known vacuolar protein. Based on their apparent molecular weights, these proteins were not CPY or PrA. Because we analyzed the medium from pulse-labeled cells, we also inferred that the rate of normal protein secretion was not grossly altered in the $v p t$ mutants. The lack of new proteins corresponding to CPY or PrA in the medium was probably due to the instability of these mislocalized proteins $(2,38)$, as well as to their relatively low abundance compared with that of the proteins in the medium observed under these experimental conditions.

In addition, many of the mutants were transformed with 


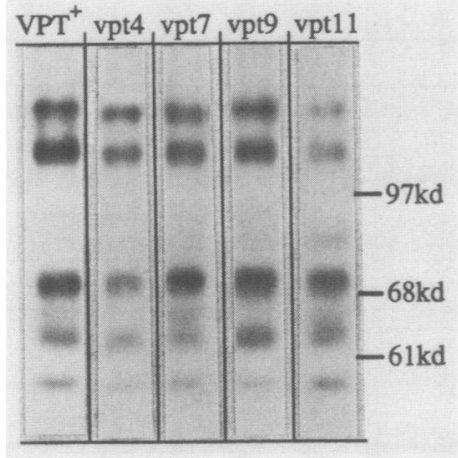

FIG. 4. Proteins secreted from wild-type and $v p t$ mutant strains. Strains were labeled with ${ }^{35} \mathrm{SO}_{4}$ for $20 \mathrm{~min}$ and chased for $20 \mathrm{~min}$ with $\left(\mathrm{NH}_{4}\right)_{2} \mathrm{SO}_{4}(1 \mathrm{mM})$. Cells were removed by centrifugation. The supernatant (medium) proteins were precipitated with trichloroacetic acid and run on a $10 \%$ polyacrylamide gel. Size standards (in kilodaltons [kd]) are as indicated.

an Inv-encoding plasmid (pRB58) and tested for normal Inv secretion (assay and calculations were as described previously [27]). The vpt mutants were found to secrete Inv at the same levels as wide-type $S$. cerevisiae. This provides further evidence that the vpt mutants carry out secretion normally.

$v p t$ mutants exhibit defects in the sorting and processing of several vacuolar proteins. To assess directly the extent of the sorting defect in the various $v p t$ mutants, we labeled and fractionated $v p t$ mutant cells. Antisera directed against CPY, $\operatorname{PrA}$, and $\operatorname{PrB}$ were used to detect the amount and form of these different vacuolar proteins in each cell fraction. Initially, cells were labeled and fractionated into medium, periplasm, and spheroplast fractions, with subsequent immunoprecipitation by anti-CPY and anti-PrA antisera. These data (not shown) allowed us to categorize the vpt mutants based on the severity of their CPY and PrA localization and processing defects. However, we noticed that during the fractionation, presumably because of the incubation time required to remove the cell wall enzymatically, some fraction of these proteins was being degraded or modified. To avoid this, we converted the mutant cells to spheroplasts before they were labeled and fractionated. In this way we were able to look at mislocalization by isolating spheroplast (pellet) and medium fractions by centrifugation. The medium fraction corresponded to all material secreted from the cells, including those proteins that are normally retained in the periplasm. A further advantage of labeling spheroplasts is that because lysed spheroplasts do not incorporate the label, it reduces the background of labeled vacuolar proteins in the medium resulting from cell lysis during spheroplast preparation. To confirm that the spheroplast-labeling technique did not alter normal protein secretion or protein sorting to the vacuole, we initially analyzed the modification and processing of the secreted enzyme (Inv) and the vacuolar proteins (CPY and PrA) in spheroplasts prepared from a wild-type strain. Both protein secretion and vacuolar protein sorting and processing appeared to be normal (Fig. 3 and 5). Indeed, we observed that the level of protein expression and the kinetics of protein modification and delivery appeared to be normal by the spheroplast-labeling technique $(8,15,18,35 \mathrm{~b})$. This argues that secretory protein traffic occurs normally in yeast spheroplasts.

Wild-type and $v p t$ mutant spheroplasts were labeled with $\left.{ }^{[35} \mathrm{S}\right]$ methionine for $20 \mathrm{~min}$ and chased with cold methionine

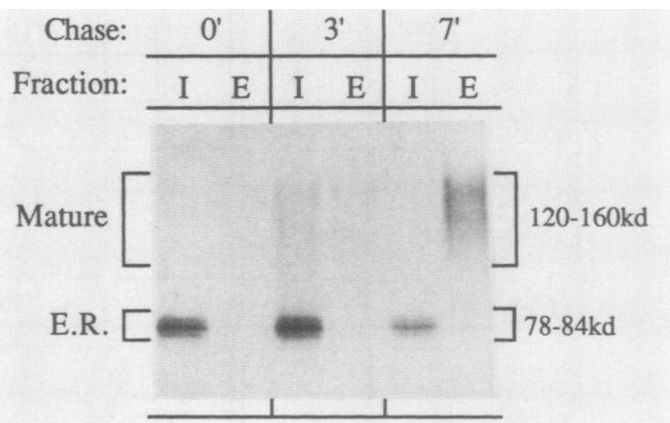

FIG. 5. Immunoprecipitation of Inv from labeled spheroplasts. VPT S. cerevisiae (SEY6211) containing the Inv-encoding plasmid pRB58 was derepressed by growth in $0.1 \%$ glucose for $30 \mathrm{~min}$ converted to spheroplasts, and labeled for $5 \mathrm{~min}$ with $\left[{ }^{35} \mathrm{~S}\right]$ methionine. Chase with cold methionine was for 0,3 , or $7 \mathrm{~min}$, as indicated. Spheroplasts were separated into pellet (I) and supernatant (E) fractions by centrifugation and immunoprecipitated with Inv antisera. The core glycosylated Inv found in the endoplasmic reticulum (E.R.) migrated as a set of bands that were converted to a heterogeneous mixture of highly glycosylated molecules in the Golgi complex prior to secretion from the cell. kd, Kilodaltons.

for $30 \mathrm{~min}$. Supernatant (external) and pellet (internal) fractions were immunoprecipitated with anti-CPY antisera. We observed two significant effects of the vpt mutants on the biogenesis of the vacuolar protein CPY (Fig. 3). First, processing was altered; 14 of the mutants exhibited no mature CPY at all (vptl, vpt3, vpt4, vpt5, vpt6, vpt7, vpt11, vpt15, vpt16, vpt17, vpt18, vpt 29 , vpt30, vpt33), and an additional $5 v p t$ mutants processed less than $30 \%$ of CPY to the mature forms (vpt8, vpt9, vpt13, vpt19, vpt26). Second, missorting and secretion of CPY were observed. Two of the $v p t$ mutants (vpt15 and vpt29) secreted more than $95 \%$ of CPY into the periplasmic and medium fraction. An additional $12 v p t$ mutants (vptl, vpt3, vpt4, vpt5, vpt6, vpt7, vpt11, vpt16, vpt17, vpt18, vpt30, vpt33) were almost as defective; they secreted between 60 and $80 \%$ of CPY (Fig. 3 ). These 14 most defective mutants exhibited a complete defect in maturation of the small amount of CPY that remained associated with the spheroplast fraction, as discussed above, indicating that the accumulated proCPY was not sorted to the vacuole or, possibly, that the vacuole of these mutants was so defective that it was not competent for the processing of proCPY. Many of the remaining vpt mutants exhibited less severe defects in the sorting of the CPY protein. In these mutants, a substantial portion of CPY remained associated with the spheroplast and was processed to the mature active enzyme, indicating that some of the proCPY was sorted correctly to the vacuole (Fig. 3).

To determine whether this protein-sorting defect is specific for CPY or extends to other vacuolar proteins, we examined sorting and processing of two additional vacuolar proteins, PrA and PrB, in the vpt mutants. Relative to CPY, lower levels of both $\operatorname{PrA}$ and $\operatorname{PrB}$ were secreted by the vpt mutants. However, we noted that the extent of the defect in maturation of these proteins paralleled that observed for CPY in each of the vpt mutants (Fig. 3 and 6). The absence of CPY in the vacuole cannot explain the accumulation of precursor forms of $\operatorname{PrA}$ and $\operatorname{PrB}$, as maturation of these proteases is not dependent on CPY function. This suggests that the defect in vacuolar sorting of $\operatorname{PrA}$ and $\operatorname{PrB}$ may be similar to that observed for CPY, but that secretion of the missorted PrA and PrB is blocked. We reasoned that the reduced level of proPrA and proPrB in the media fraction 


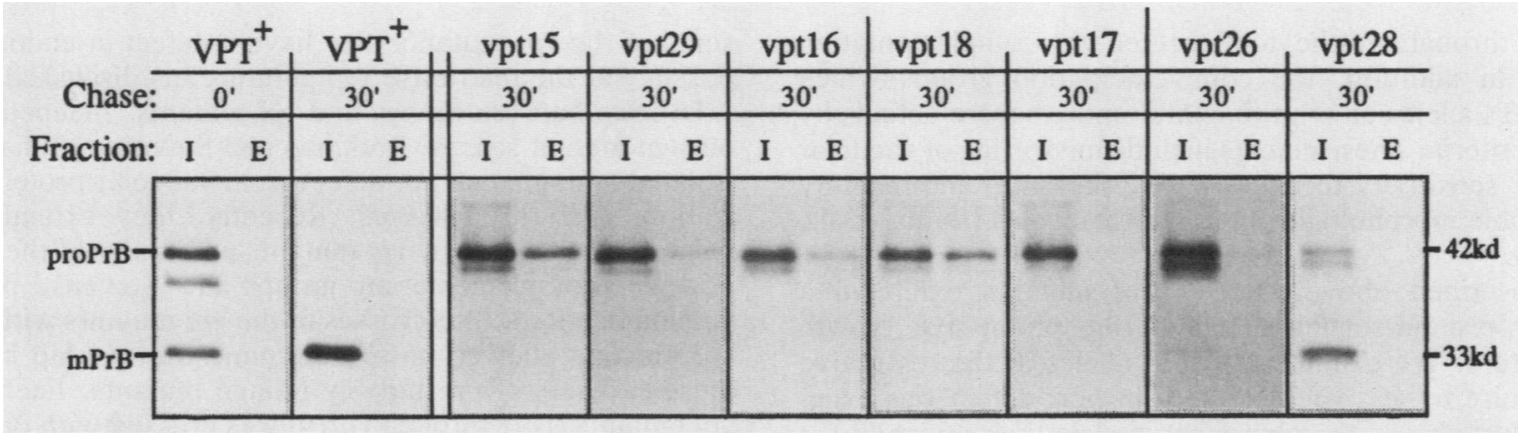

FIG. 6. Immunoprecipitation of PrB in wild-type (SEY6210 and SEY6211) and vpt mutant yeast strains. Spheroplasts were labeled as described in the text and chased for the indicated times (in minutes). I, Internal (spheroplast pellet) fraction; E, external (supernatant) fraction. PrB antiserum was used for the immunoprecipitations. The migration positions of precursor (proPrB) and mature (mPrB) forms of $\operatorname{PrB}$ and their approximate molecular sizes (in kilodaltons [kd]) are indicated. Note the extra band at 0 min; presumably, it was an intermediate in $\operatorname{PrB}$ maturation.

might be due to a reduced rate in secretion of these missorted proteins. To address this possibility, we extended the length of the chase period from 30 to $90 \mathrm{~min}$ and otherwise performed the fractionation and immunoprecipitation procedures as described above. When this experiment was carried out on vpt5, vpt11, and vpt15, no increases in the levels of proPrA or proPrB were observed in the medium after this extended chase period (data not shown). In this experiment we also observed that no additional processing of the proPrA and proPrB occurred during the chase.

An alternative explanation is that the lack of these proteins in the medium fraction might not be due to a block in secretion of the mislocalized zymogens, but rather to the specific or nonspecific association of these proteins with the spheroplast membrane surface. To test this possibility, we treated the labeled spheroplasts with $500 \mu \mathrm{g}$ of proteinase $\mathrm{K}$ per $\mathrm{ml}$ for $30 \mathrm{~min}$ at $0^{\circ} \mathrm{C}$ to determine whether any of the cell-associated proPrA and proPrB was accessible to the exogenously added protease. Several $v p t$ mutants (vpt5, $v p t 17$, and vpt26) were analyzed in this way, and in each case, $\operatorname{Pr} A$ and $\operatorname{PrB}$ were unaffected by the addition of proteinase $\mathrm{K}$. As expected, secreted CPY was degraded under these conditions. Both $\operatorname{PrA}$ and $\operatorname{PrB}$ were, however, subject to degradation if the detergent Triton X-100 (1\%) was added prior to protease addition. Taken together, these experiments indicate that proPrA and proPrB accumulate in some intracellular compartment. The present data do not rule out the vacuole as the site in which these proteins reside; however, the lack of processing of the accumulated proteins suggests that this may not be case. This lack of processing does not eliminate the vacuole as the site of accumulation of PrA and PrB, because of the observation that in missense PrA mutants, CPY activity is normal but $\operatorname{PrA}$ activity is decreased (23).

Several $v p t$ mutants are temperature sensitive for growth. It is not clear whether vacuolar protein sorting is essential for yeast cell growth. The pep4 mutants (45) are defective in enzyme activities of many soluble vacuolar hydrolases, yet they grow normally at all temperatures. We selected our mutants at $26^{\circ} \mathrm{C}$ to allow us to score for potential temperature-conditional phenotypes. Thus, after the mutants were selected for their ability to grow on sucrose-containing medium at $26^{\circ} \mathrm{C}$, they were tested for their ability to grow at $37^{\circ} \mathrm{C}$ on YPD medium. Of the 505 mutants isolated, 23 (approximately 5\%) were Ts for growth at $37^{\circ} \mathrm{C}$ (i.e., they did not form colonies at $37^{\circ} \mathrm{C}$ ). These Ts mutants belonged to six vpt complementation groups (vpt11, vpt15, vpt16, vpt18, $v p t 29$, and $v p t 33)$. In addition, one allele of $v p t 3$ had a partial Ts phenotype (very small colonies at $37^{\circ} \mathrm{C}$ ). Genetic crosses and tetrad analysis were used to confirm that in each case the Ts phenotype cosegregated with the vpt protein sorting defect. One Ts allele from each complementation group was crossed to the parent strain (SEY6210 or SEY6211), sporulated, and subjected to tetrad dissection. Ten or more tetrads were examined for each cross (an example of the cosegregating phenotypes is shown in Fig. 7). The remaining Ts alleles in a given complementation group were inferred to be linked to the appropriate vpt locus by complementation testing at $37^{\circ} \mathrm{C}$. It is interesting that of the $v p t$ complementation groups that contained Ts alleles (not including vpt3), 22 of 50 total alleles (i.e., 44\%) in these complementation groups were Ts. Thus, the Ts alleles are not scattered at

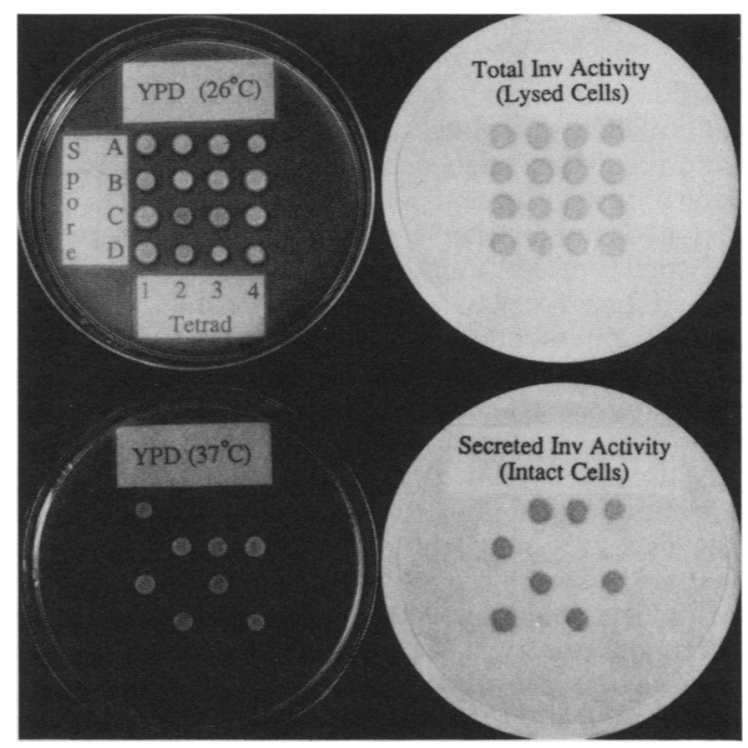

FIG. 7. Demonstration that the Ts growth phenotype and the vpt mislocalization phenotype cosegregate in four representative tetrads from a cross of a Ts allele of vpt16 with strain SEY6210. The filter assay of the lysed segregant patches showed that each spore had Inv activity. Below, the Inv filter assay of intact segregant patches showed that the ability to secrete Inv activity (i.e., the CPY-Inv hybrid protein) segregated 2:2. Similar cosegregation of the Ts and $v p t$ defects was seen for $v p t 3, v p t 11, v p t 15, v p t 18, v p t 29$, and $v p t 33$. At least 10 tetrads were analyzed in each case. 
random throughout the total set of $v p t$ complementation groups. In addition, the complementation groups which possess Ts alleles all tend to exhibit more severe defects by several criteria. These defects include missorting of vacuolar proteins, sensitivity to high osmotic pressure, and inability to assemble morphologically normal vacuoles (Banta et al., in press).

As described above, the Ts vpt mutants exhibited a vacuolar protein-sorting defect at the permissive growth temperature. We examined the Ts alleles at the restrictive temperature to see whether the transport defect was exaggerated further and thereby might explain their inviability at $37^{\circ} \mathrm{C}$. The mutants were preincubated for $60 \mathrm{~min}$ at $37^{\circ} \mathrm{C}$ and then labeled for $20 \mathrm{~min}$ with ${ }^{35} \mathrm{SO}_{4}$ and chased for $20 \mathrm{~min}$, both at $37^{\circ} \mathrm{C}$. Immunoprecipitation with CPY antibody showed that there was no apparent difference in CPY processing or secretion at the restrictive temperature (data not shown), but it should be noted that all of these Ts $v p t$ mutants exhibited extreme defects in vacuolar protein sorting, even at the permissive temperature. Since the Ts vpt mutants secreted Inv at $37^{\circ} \mathrm{C}$, secretion apparently is not blocked at this temperature (see below). Because the defect in CPY sorting and processing was nearly complete even at the permissive growth temperature, the absence of growth at the nonpermissive temperature may be explained by the appearance of a more complete sorting defect at the high temperature, which alters the sorting of additional vacuolar proteins and possibly also affects secreted proteins that are essential for growth. Alternately, it may be that the combined stress of growth at a high temperature together with a defect in vacuolar protein sorting may, in an additive fashion, prevent cell growth at $37^{\circ} \mathrm{C}$. For example, it is possible that a loss of vacuole protein localization at a high temperature may result in a block in secretion or endocytosis, or both, since these defects have been shown to be lethal (27, 34). To address these questions, the Ts vpt mutants were tested in several ways for possible secretory or endocytic defects. First, the Ts $v p t$ mutants were transformed with a plasmid (pRB58) carrying the entire wild-type structural gene and regulatory sequences of $S U C 2$ and tested for Inv secretion at restrictive and permissive temperatures. For the experiment at the restrictive temperature, cells were shifted to $37^{\circ} \mathrm{C}$ for 60 or $120 \mathrm{~min}$ prior to the assay. Activities in whole and lysed cells were measured and compared to determine the percentage of Inv secreted (27). The mutants that were tested were not found to be significantly different from the parental strain SEY6210 harboring the same SUC2 plasmid (data not shown). This implies that the Ts $v p t$ mutants do not exhibit an Inv secretion defect at $37^{\circ} \mathrm{C}$. Second, the Ts $v p t$ mutants were tested for genetic overlap with the Ts secretion-defective mutants secl-sec23 (27) and $\sec 53(10)$. The sec mutants are defective for the secretion of Inv at $37^{\circ} \mathrm{C}$ and are blocked for transport at various stages of the secretory pathway in $S$. cerevisiae (26). sec $\times v p t$ diploid strains were selected and tested for complementation of the temperature sensitivity at $37^{\circ} \mathrm{C}$. In each case complementation was observed, implying that there is no genetic overlap between these two sets of mutants. The Ts vpt mutants were also crossed with the Ts endocytosis-defective mutant endl (5). Diploid strains were again tested for Ts growth defects. It was found that vpt 11 did not complement endl, implying that these two mutants are allelic to one another. In addition, the ENDI gene (a gift from $\mathrm{H}$. Riezman) was found to rescue the Ts defect of the vptll allele tested (P. Herman, unpublished data). This genetic overlap raises the possibility that some of the vpt mutants may have a defect in endocytosis, possibly at the restrictive temperature, as discussed above.

Overlap between the $v p t$ and $v p l$ mutants. Independent of our vpt mutant screen, Rothman and Stevens (35) have also isolated mutants that are defective in vacuolar protein localization, vpll through vpl8. Recently, they extended this work and selected new $v p l$ mutants and assigned them to 11 new $v p l$ complementation groups (T. Stevens, personal communication). Intercrosses of the vpt mutants with all the $v p l$ mutants allowed us to determine the overlap between these two sets of presumably related mutants. Each of the $v p l$ mutants (vpll through vpll9) was crossed with our Suc ${ }^{-}$ parental strain, diploid strains were selected and sporulated, and $\mathrm{Suc}^{-} v p l$ segregants were identified. These $\Delta s u c v p l$ mutants were then crossed to representative vpt mutant alleles from each complementation group, which harbored the CPY-Inv433 hybrid protein-encoding plasmid. Mislocalization of the hybrid protein from the diploid strains was scored by Inv filter assays. The results of these complementation studies are shown in Table 3. We observed that 12 of the 33 vpt mutants overlapped with $v p l$ complementation groups. This overlap was confined in most cases to $v p t$ complementation groups with the less severe vacuolar protein localization defects (Table 1 and 3). At present, we do not have an explanation for this observation. Differences in the selection schemes used or in the starting strains may have affected in part the final set of mutants obtained. J. H. Rothman and T. H. Stevens (unpublished data) have used another complementation assay technique and have independently confirmed each of these results. To avoid future confusion regarding these two classes of mutants, we have decided to use the general term vps for vacuolar protein sorting to describe both classes of mutants (Table 3).

Some differences were seen between $v p l$ and $v p t$ mutants in terms of the levels of missorting of vacuolar proteins. For example, $v p t 5$ and $v p l 5$ were in the same complementation group (vps5). The vpl5 allele analyzed by Rothman and Stevens (35) showed a weaker protein sorting defect for CPY and PrA than did vpt5 (2) (Fig. 3). This result may indicate allele differences. However, since the two mutations were in unrelated genetic backgrounds, the discrepancy also could be due to strain background differences.

Another class of pleiotropic mutants, the pep mutants, also has been shown to affect vacuolar protein processing $(16,17)$. Complementation analysis has shown that there is genetic overlap between subsets of the pep, vpt, and $v p l$ mutants (T. Stevens and E. Jones, personal communication). The overlap between the $v p t$ and pep mutants is as follows: $v p t 3=$ pep $21, v p t 4=$ pep $8, v p t 5=$ pep10, vpt $11=$ pep5, vpt13 = pep12, and vpt17 = pep6.

\section{DISCUSSION}

In an effort to identify additional mutants that exhibit novel defects in vacuolar protein sorting and to develop an appreciation for the number of gene functions that may contribute to this process, we exploited a CPY-Inv fusionbased selection scheme (2) to isolate more than 500 new $v p t$ mutants. The mutants were assigned to 33 different complementation groups, 7 of which contained Ts alleles. Even though this was an unexpectedly large number of complementation groups, our data suggest that many additional gene functions are likely to influence the vacuolar protein delivery pathway. Several of the newly isolated $v p t$ mutants could not be assigned to any of these 33 complementation groups. In addition, genetic comparison of the vpt mutants 
TABLE 3. Complementation analysis of $v p t$ and $v p l$ mutants

\begin{tabular}{|c|c|c|}
\hline $\begin{array}{c}v p t \\
\text { mutant }\end{array}$ & $\begin{array}{c}v p l \\
\text { mutant }\end{array}$ & $\begin{array}{l}\text { Common complementation } \\
\text { group name }\end{array}$ \\
\hline vpt26 & vpll & vpsl \\
\hline & $v p l 2$ & vps 2 \\
\hline vpt17 & vpl3 & vps3 \\
\hline vpt 10 & vpl4 & vps 4 \\
\hline$v p t 5$ & vpl5 & vps5 5 \\
\hline \multirow[t]{2}{*}{ vpt13 } & vpl6 & vps6 \\
\hline & vpl7 & vps 7 \\
\hline vpt8 & \multirow[t]{3}{*}{ vplo } & vps8 \\
\hline vpt9 & & $v p s 9$ \\
\hline vptl & & vps 10 \\
\hline vptll & \multirow[t]{9}{*}{$v p l 9$} & vps 11 \\
\hline vpt 12 & & vps 12 \\
\hline vpt2 & & vps 13 \\
\hline vpt 14 & & vps 14 \\
\hline vpt 15 & & vps 15 \\
\hline vpt 16 & & vps 16 \\
\hline vpt3 & & vps 17 \\
\hline vpt 18 & & vps 18 \\
\hline vpt 19 & & vps 19 \\
\hline vpt20 & \multirow[t]{2}{*}{ vpllo } & vps 20 \\
\hline vpt21 & & vps 21 \\
\hline vpt22 & vpll4 & vps 22 \\
\hline vpt 23 & \multirow[t]{2}{*}{ vplls } & vps 23 \\
\hline vpt24 & & vps 24 \\
\hline vpt 25 & \multirow[t]{3}{*}{ vpll2 } & vps 25 \\
\hline vpt4 & & vps 26 \\
\hline vpt27 & & vps27 \\
\hline vpt28 & \multirow[t]{8}{*}{ vpll3 } & vps 28 \\
\hline vpt6 & & vps 29 \\
\hline vpt 30 & & vps 30 \\
\hline vpt31 & & vps31 \\
\hline vpt32 & & vps32 \\
\hline vpt33 & & vps33 \\
\hline vpt29 & & vps34 \\
\hline \multirow[t]{6}{*}{ vpt7 } & & vps35 \\
\hline & vplll & vps36 \\
\hline & vpll6 & vps37 \\
\hline & vpll7 & vps38 \\
\hline & vplls & vps39 \\
\hline & vplls & vps 40 \\
\hline
\end{tabular}

a The vpt mutants were crossed with the $v p l$ mutants (that had been made $\Delta s u c 2$ by crossing them with strain SEY6210) and tested for their ability to complement the CPY-Inv433 sorting defect. Extensive overlapping was seen, and it is proposed that to avoid confusion in the future, these strains should be referred to as vps mutants (for vacuolar protein sorting) and the numbers should be as listed here (see also J. H. Rothman and T. H. Stevens, manuscript in preparation).

with similar mutants from other laboratories (sec, pep, vpl, end $[5,10,16,27,35])$, affecting secretory protein delivery or processing, indicated that although there was overlap among some of these different sets of mutants, more than 50 gene functions are implicated in the vacuolar protein sorting process.

This large number of genes can be interpreted to indicate that vacuolar protein sorting is a very complex process involving a series of distinct and precisely controlled sorting events. These include translocation into the endoplasmic reticulum, sorting to and through the Golgi complex, separation from proteins destined for secretion or Golgi complex retention, and selective delivery to the vacuole, possibly via an intermediate endosomelike compartment. Because each of these steps is likely to be catalyzed by proteins that are reused through several rounds of protein transfer, any mutations that affect their level of expression, stability, activity, or ability to recycle back to the appropriate compartment of action may lead to sorting defects. Alternatively, the large number of mutants identified may suggest that many unrelated physiological and cellular defects can interfere with vacuolar protein sorting by indirectly influencing the process. That the former argument (that the $v p t$ mutations cause specific changes in the targeting machinery) may indeed be the case for the more defective vpt mutants is supported by the following observations. (i) All of the late-acting sec mutants, which exhibited major defects in protein secretion, did not alter the delivery or maturation of CPY (38). (ii) Yeast strains deleted for the gene encoding the clathrin heavy chain did not exhibit defects in vacuolar protein biogenesis, even though the growth rate and intracellular morphology of these mutants were dramatically different from those of wild-type cells $(29,30)$. (iii) Cell fractionation and enzyme assay data ruled out the release of vacuolar proteins as a consequence of yeast cell lysis. (iv) Though many of the vpt mutants exhibited major defects in the targeting and maturation of vacuolar proteins, protein secretion as well as many other cell functions, including cell division, mating, and respiratory growth, remained largely unaffected. Direct evidence concerning the specificity of the events affected by the $v p t$ mutants depends on the identification of the various VPT gene products, their site of action in the cell, and their individual biochemical activities.

Several observations led us to suspect that if vacuolar enzymes were not efficiently targeted to the vacuole, they would appear in the medium. Both CPY and PrA travel through the initial part of the secretory pathway (38, Klionsky et al., in press), so it can be postulated that if CPY or PrA fails to be diverted to the vacuole at the appropriate step, they might continue to be carried through the secretory pathway and end up at the cell surface. Indeed, much evidence has accumulated suggesting that many proteins are secreted by a default mechanism, while vacuolar and lysosomal enzymes must be actively sorted away from these proteins $(4,31)$. In addition, gene dosage-induced overproduction of CPY or PrA or mutational changes in the vacuolar sorting signals of these proteins lead to their missorting and secretion $(15,18 a, 39,41)$, suggesting that if the vacuolar targeting apparatus is overloaded or unable to recognize vacuolar proteins, the default reaction for these proteins is secretion. A similar overproduction-induced mislocalization phenotype has been observed for the vacuolar CPY-Inv and PrA-Inv hybrid proteins $(2,18 \mathrm{a})$. Finally, an initial set of vacuolar protein mislocalization mutants which missort and secrete vacuolar enzymes has already been obtained by this scheme $(2,35)$.

The vpt mutants were selected on the basis of their ability to missort and secrete a hybrid vacuolar protein, CPY-Inv. The sorting of the artificial hybrid protein CPY-Inv fairly accurately reflects the sorting of the native CPY protein. Several lines of evidence support this conclusion. CPY-Inv hybrid proteins, carrying the CPY sorting signal, are very efficiently localized to the vacuole, as is true for native CPY $(15,18 a)$. Mutants selected as defective in the vacuolar sorting of CPY-Inv hybrid proteins ( $v p t$ ) also exhibit similar defects in the sorting of wild-type CPY (2). Enzyme assay data from this and previous studies (2) demonstrate that the extent of mislocalization of both the CPY-Inv hybrid proteins and native CPY protein correlate closely in each of the $v p t$ mutants (Table 2). The vpt mutants genetically overlapped with the $v p l$ mutants, which were selected by an independent scheme for defects in CPY sorting (35) (Table $3)$. The gene fusion approach also offers the advantage that it can be generally applied to the study of other gene products 
independent of any knowledge of their biochemical activity $(6,7,14)$.

It is of interest that although the extents of the sorting defects seen in different $v p t$ mutants may vary, each mutant individually appears to exhibit qualitatively similar defects in the sorting and processing of the three proteins that were analyzed: CPY, PrA, and PrB. Although the vpt mutants did not secrete the same level of proCPY, proPrA, or proPrB, they all appeared to exhibit similar proteolytic processing defects for each of these proteins (Fig. 3 and 6). The vpt mutants caused significantly higher levels of proCPY to be mislocalized to the medium than the levels observed for either proPrA or proPrB. Most of the vpt mutants secreted p2CPY forms, indicating that this protein passes correctly through the Golgi complex. Although some level of secretion of proPrA and proPrB was observed in many of the $v p t$ mutants, the bulk of each of these precursor proteins appeared to accumulate in an intracellular compartment distinct from the vacuole. They remained associated with the spheroplast cell fraction and were not degraded when extracellular proteases were added to the labeled spheroplasts. The proteins accumulated in their precursor forms, even though in many $v p t$ mutants mature forms were also present, indicating that the vacuoles of these mutants were still competent for precursor maturation (Fig. 3 and 6). We do not know where in the cells the precursors accumulated or why they were not secreted like proCPY. The PrA-Inv hybrid protein was efficiently secreted by many of the $v p t$ mutants (Table 1), suggesting that PrA itself is missorted in the mutants but cannot transit through later stages of the secretory pathway as efficiently as the PrA-Inv hybrid protein can. (Inv is normally secreted by $S$. cerevisiae and may facilitate transit of the PrA-Inv hybrid.) proPrA and proPrB may associate specifically or nonspecifically with some component of an intracellular membrane, or they may be diverted into a compartment that does not communicate with the cell surface. The vacuolar protein-sorting signals in both proCPY and proPrA have been identified, but they appear to share no significant primary sequence similarities $(15,18,41)$ and therefore may be recognized by different carrier systems.

An important unresolved question regarding protein sorting to mammalian lysosomes or to the yeast vacuole is whether luminal proteins transit together with integral membrane proteins, and if so, does a common sorting apparatus participate in directing the delivery of these two distinct sets of proteins? In the case of I-cell disease, many lysosomal luminal enzymes are missorted; however, certain membrane proteins continue to be faithfully sorted to the vacuole (33, $37,40)$. When we assayed for the location of the vacuolar membrane marker enzyme $\alpha$-mannosidase, we found that it was not mislocalized to the cell surface in most of the vpt mutants. Vacuoles isolated from two of these mutants (vpt3 and vpt4) retained normal levels of this enzyme activity (2). However, a small subset of the vpt mutants did exhibit significant defects in the sorting of $\alpha$-mannosidase activity. Alleles of vpt11, vpt16, vpt18, and vpt33 secreted up to $50 \%$ of their $\alpha$-mannosidase activity to the cell surface. Interestingly, this same set of vpt mutants also exhibited other phenotypes that were consistent with the fact that these mutants have defects in both luminal and membrane vacuolar protein sorting. These phenotypes include the absence of a detectable vacuole in the mutant cells, as determined by light or electron microscopy, and accumulation of novel membrane-enclosed structures that may correspond to intermediates in vacuole membrane biogenesis (Banta et al., in press). These results indicate that at least some vacuolar membrane proteins may share a common sorting step or compartment with soluble vacuolar proteins en route to the vacuole.

A useful observation is that seven of the vpt complementation groups had alleles exhibiting a Ts growth defect (on YPD medium at $37^{\circ} \mathrm{C}$ ). In each case, this Ts growth defect was shown to be genetically linked to the $v p t$ phenotype. It is probably not coincidental that these mutants all exhibited interesting additional vacuole-related defects. These Ts vpt mutants could be divided into three groups on the basis of their additional phenotypes and characteristics. (i) vpt 15 and $v p t 29$ were the two complementation groups with the most severe localization defect for CPY and PrA (Fig. 3). In addition, they both exhibited abnormal morphologies, including the accumulation of vesicular and Golgi complexrelated structures, and apparently enlarged vacuoles relative to wild-type $S$. cerevisiae (Banta et al., in press). (ii) vptll, vpt 16, vpt 18, and vpt33 also exhibited severe defects in the sorting of CPY and showed the accumulation of most of their PrA in the precursor form, indicating that it probably also did not reach the vacuole. These mutants exhibited the most striking structural defects of any of the vpt mutants, since they did not contain a morphologically identifiable vacuole. (iii) vpt3 had one Ts allele and was moderately defective in CPY and PrA localization, as assessed by immunoprecipitation (Fig. 3). The vpt3 mutant is interesting morphologically because it appears to contain highly fragmented vacuoles (Banta et al., in press).

One of the vpt mutants (vptll) was found to be allelic to the Ts endocytosis-defective mutant endl (5). This observation is interesting because it provides the first genetic evidence for a link between the endocytotic and biosynthetic delivery routes to the yeast vacuole. This result was not unexpected, because some mutants defective in the secretory pathway ( $\mathrm{sec}$ ) are also defective in endocytosis (34), and as discussed above, early stages of vacuolar protein targeting are sec dependent (38). It will be of interest to address the endocytic competence of the vpt mutants.

We identified many new genes whose products are involved in the process of vacuolar protein targeting. We expect that many of the gene products act directly in the targeting and transport of molecules to the yeast vacuole. More detailed information about how the vacuolar targeting pathway operates is anticipated on cloning of the VPT genes and detailed biochemical characterization of the gene products.

\section{ACKNOWLEDGMENTS}

We thank John C. L. DeModena for excellent technical assistance and Cathy Elkins for patience in typing the manuscript. We are grateful to Elizabeth Jones and Charles Moehle for the generous gift of anti-PrB antisera and Howard Riezman, Joel Rothman, and Tom Stevens for communicating their results prior to publication. In addition, we thank David Bedwell, Paul Herman, and Tom Vida for critical reading of the manuscript and valuable discussions during the course of the work.

This study was supported by Public Health Service grant GM32703 from the National Institutes of Health (to S.D.E.). J.S.R. was supported by graduate fellowships from the Lucy Mason Clark fund and the Markey Charitable Trust fund, L.M.B. was supported by graduate fellowships from the National Science Foundation and General Electric Co. (Schenectady, N.Y.), D.J.K. was supported by a research fellowship from the Helen Hay Whitney Foundation, and S.D.E. is an NSF Presidential Young Investigator supported by grant DCB-8451633 from the National Science Foundation. 


\section{LITERATURE CITED}

1. Ammerer, G., C. P. Hunter, J. H. Rothman, G. C. Saari, L. A. Valls, and T. H. Stevens. 1986. PEP4 gene of Saccharomyces cerevisiae encodes proteinase A, a vacuolar enzyme required for processing of vacuolar precursors. Mol. Cell. Biol. 6:2490 2499.

2. Bankaitis, V. A., L. M. Johnson, and S. D. Emr. 1986. Isolation of yeast mutants defective in protein targeting to the vacuole. Proc. Natl. Acad. Sci. USA 83:9075-9079.

3. Bernstein, M., W. Hoffman, G., Ammerer, and R. Schekman. 1985. Characterization of a gene product (Sec53p) required for protein assembly in the yeast endoplasmic reticulum. J. Cell Biol. 101:2374-2382.

4. Burgess, T. L., and R. B. Kelly. 1987. Constitutive and regulated secretion of proteins. Annu. Rev. Cell Biol. 3:243-293.

5. Chvatcho, Y., I. Howald, and H. Riezman. 1986. Two yeast mutants defective in endocytosis are defective in pheromone response. Cell 46:355-364.

6. Douglas, M. G., B. L. Geller, and S. D. Emr. 1984. Intracellular targeting and import of an $F_{1}$-ATPase $\beta$-subunit- $\beta$-galactosidase hybrid protein into yeast mitochondria. Proc. Natl. Acad. Sci. USA 81:3983-3987.

7. Emr, S. D., R. Schekman, M. C. Flessel, and J. Thorner. 1983. An MFol-SUC2( $\alpha$-factor invertase) gene fusion for study of protein localization and gene expression in yeast. Proc. Natl. Acad. Sci. USA 80:7080-7084.

8. Esmon, P. C., B. E. Esmon, T. E. Schauer, A. Taylor, and R. Schekman. 1987. Structure, assembly, and secretion of octameric invertase. J. Biol. Chem. 262:4387-4394.

9. Farquhar, M. G., and G. E. Palade. 1981. The Golgi apparatus (complex)-(1954-1981)-from artifact to center stage. J. Cell Biol. 81:77s-103s.

10. Ferro-Novick, S., P. Novick, C. Field, and R. Schekman. 1984. Yeast secretory mutants that block the formation of active cell surface enzymes. J. Cell Biol. 98:35-43.

11. Griffiths, F., and K. Simons. 1986. The trans Golgi network: sorting at the exit site of the Golgi complex. Science 234:438-443.

12. Hasilik, A., and W. Tanner. 1978. Biosynthesis of the vacuolar yeast glycoprotein carboxypeptidase Y. Eur. J. Biochem. 85: $599-608$.

13. Hemmings, B. A., G. S. Zubenko, A. Hasilik, and E. W. Jones. 1981. Mutant defective in processing of an enzyme located in the lysosome-like vacuole of Saccharomyces cerevisiae. Proc. Natl. Acad. Sci. USA 78:435-439.

14. Hofimann, W. 1987. CANI-SUC2 gene fusion studies in Saccharomyces cerevisiae. Mol. Gen. Genet. 210:277-281.

15. Johnson, L. M., V. A. Bankaitis, and S. D. Emr. 1987. Distinct sequence determinants direct intracellular sorting and modification of a yeast vacuolar protease. Cell 48:875-885.

16. Jones, E. W. 1977. Proteinase mutants of Saccharomyces cerevisiae. Genetics 85:23-33.

17. Jones, E. W., G. S. Zubenko, and R. R. Parker. 1982. PEP4 gene function is required for expression of several vacuolar hydrolases in Saccharomyces cerevisiae. Genetics 102:665-677.

18. Klionsky, D. J., L. M. Banta, and S. D. Emr. 1988. Intracellular sorting and processing of a yeast vacuolar hydrolase: proteinase A propeptide contains vacuolar targeting information. Mol. Cell. Biol. 8:2105-2116.

18a.Klionsky, D. J., L. M. Banta, J. S. Robinson, and S. D. Emr. 1988. Vacuolar protein targeting and organelle biogenesis in yeast, p. 173-186. In R. Bradshaw, L. McAlister-Henn, and M. Douglas (ed.), Molecular biology of intracellular protein sorting and organelle assembly. Alan R. Liss, Inc., New York.

19. Kornfeld, S. 1986. Trafficking of lysosomal enzymes in normal and diseased states. J. Clin. Invest. 77:1-6.

20. Matile, P., H. Moor, and C. F. Robinow. 1969. Yeast cytology, p. 219-302. In A. H. Rose and J. S. Harrison (ed.), The yeasts. Academic Press, Inc., London.

21. Mechler, B., H. H. Hirsch, H. Müller, and D. H. Wolf. 1988. Biogenesis of the yeast lysosome (vacuole): biosynthesis and maturation of proteinase yscB. EMBO J. 7:1705-1710.
22. Mechler, B., M. Müller, H. Müller, F. Meussdoerfier, and D. H. Wolf. 1982. In vivo biosynthesis of the vacuolar proteinases A and B in the yeast Saccharomyces cerevisiae. J. Biol. Chem. 257:11203-11206.

23. Mechler, B., H. Müller, and D. H. Wolf. 1987. Maturation of vacuolar (lysosomal) enzymes in yeast: proteinase yscA and proteinase yscB are catalysts of the processing and activation event of carboxypeptidase yscY. EMBO J. 6:2157-2163.

24. Moir, D., S. Stewart, B. Osmond, and D. Botstein. 1982. Coldsensitive cell-division-cycle mutants of yeast: isolation, properties and pseudoreversion studies. Genetics 100:547-563.

25. Mortimer, R. K., and D. C. Hawthorne. 1986. Genetic mapping in Saccharomyces. Genetics 53:165-173.

26. Novick, P., S. Ferro, and R. Schekman. 1981. Order of events in the yeast secretory pathway. Cell 25:461-469.

27. Novick, P., C. Field, and R. Schekman. 1980. Identification of 23 complementation groups required for post-translational events in the yeast secretory pathway. Cell 21:205-215.

28. Opheim, D. J. 1978. $\alpha$-D-Mannosidase of Saccharomyces cerevisiae, characterization and modulation of activity. Biochim. Biophys. Acta 524:121-130.

29. Payne, G. S., D. Baker, E. van Tuinen, and R. Schekman. 1988. Protein transport to the vacuole and receptor-mediated endocytosis by clathrin heavy chain-deficient yeast. J. Cell. Biol. 106: 1453-1461.

30. Payne, G. S., T. B. Hasson, M. S. Hasson, and R. Schekman. 1987. Genetic and biochemical characterization of clathrindeficent Saccharomyces cereivisiae. Mol. Cell. Biol. 7:3888-3898.

31. Pfeffer, S. R., and J. E. Rothman. 1987. Biosynthetic protein transport and sorting by the endoplasmic reticulum and Golgi. Annu. Rev. Biochem. 56:829-852.

32. Reid, G. A. 1983. Pulse labeling of yeast cells and spheroplasts. Methods Enzymol. 97:324-329.

33. Reitman, M. L., A. Varki, and S. Kornfeld. 1981. Fibroblasts from patients with I-cell disease and pseudo-Hurler polydystrophy are deficient in uridine $5^{\prime}$-diphosphate- $N$-acetylglucosamine:glycoprotein $N$-acetylglucosaminylphosphotransferase activity. J. Clin. Invest. 67:1574-1579.

34. Riezman, H. 1985. Endocytosis in yeast: several of the yeast secretory mutants are defective in endocytosis. Cell 40:10011009.

35. Rothman, J. H., and T. H. Stevens. 1986. Protein sorting in yeast: mutants defective in vacuole biogenesis mislocalize vacuolar proteins into the late secretory pathway. Cell 47:10411051.

35a.Rothman, J. H., and T. H. Stevens. 1988. Protein sorting and biogenesis of the lysosome-like vacuole in yeast, p. 317-362. In R. C. Das and P. W. Robins (ed.), Protein transfer and organelle biogenesis. Academic Press, San Diego, Calif.

35b.Schauer, I., S. Emr, C. Gross, and R. Schekman. 1985. Invertase signal and mature sequence substitutions that delay intercompartmental transport of active enzymes. J. Cell Biol. 100:1664 1675.

36. Sherman, F., G. R. Fink, and C. W. Lawrence. 1979. Methods in yeast genetics: a laboratory manual. Cold Spring Harbor Laboratory, Cold Spring Harbor, N.Y.

37. Sly, W. S., and H. D. Fischer. 1982. The phosphomannosyl recognition system for intracellular and intercellular transport of lysosomal enzymes. J. Cell. Biochem. 18:67-85.

38. Stevens, T., B. Esmon, and R. Schekman. 1982. Early stages in the yeast secretory pathway are required for transport of carboxypeptidase $Y$ to the vacuole. Cell 30:439-448.

39. Stevens, T. H., J. H. Rothman, G. S. Payne, and R. Schekman. 1986. Gene dosage-dependent secretion of yeast vacuolar carboxypeptidase Y. J. Cell. Biol. 102:1551-1557.

40. Tager, J. M. 1985. Biosynthesis and deficiency of lysosomal enzymes. Trends Biochem. Sci. 10:324-326.

41. Valls, L. A., C. P. Hunter, J. H. Rothman, and T. H. Stevens. 1987. Protein sorting in yeast: the localization determinant of yeast vacuolar carboxypeptidase $\mathrm{Y}$ resides in the propeptide. Cell 48:887-897.

42. Wickerham, L. J. 1946. A critical evaluation of the nitrogen 
assimilation tests commonly used in the classification of yeasts. J. Bacteriol. 52:293-301.

43. Wiemken, A., M. Schellenberg, and K. Urech. 1979. Vacuoles: the sole compartments of digestive enzymes in yeast (Saccharomyces cerevisiae)? Arch. Microbiol. 123:23-35.

44. Yoshihisa, T., Y. Otsumi, and Y. Anraku. 1988. Solubilization and purification of vacuolar membranes in Saccharomyces cerevisiae. J. Biol. Chem. 263:5158-5163.

45. Zubenko, G. S., F. J. Park, and E. W. Jones. 1983. Mutations in PEP4 locus of Saccharomyces cerevisiae block final step in maturation of two vacuolar hydrolases. Proc. Natl. Acad. Sci. USA 80:510-514. 